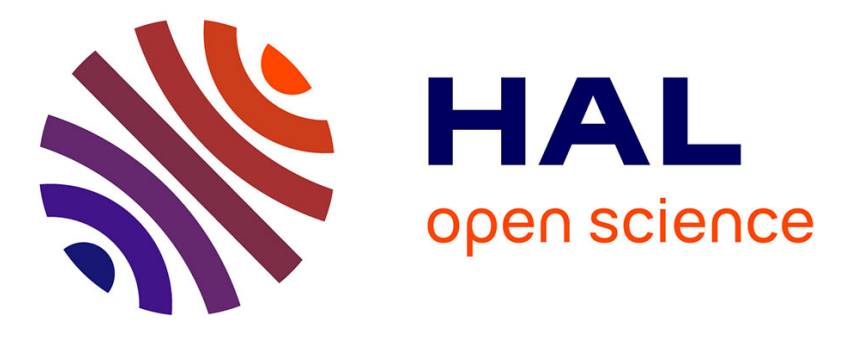

\title{
Evolution des extrêmes hydrométriques en France à partir de données observées
}

Benjamin Renard, M. Lang, P. Bois, A. Dupeyrat, O. Mestre, H. Niel, J. Gailhard, C. Laurent, L. Neppel, Eric Sauquet

\section{To cite this version:}

Benjamin Renard, M. Lang, P. Bois, A. Dupeyrat, O. Mestre, et al.. Evolution des extrêmes hydrométriques en France à partir de données observées. La Houille Blanche - Revue internationale de l'eau, 2006, 6, p. 48 - p. 54. 10.1051/lhb:2006100 . hal-00451696

\section{HAL Id: hal-00451696 https://hal.science/hal-00451696}

Submitted on 29 Jan 2010

HAL is a multi-disciplinary open access archive for the deposit and dissemination of scientific research documents, whether they are published or not. The documents may come from teaching and research institutions in France or abroad, or from public or private research centers.
L'archive ouverte pluridisciplinaire HAL, est destinée au dépôt et à la diffusion de documents scientifiques de niveau recherche, publiés ou non, émanant des établissements d'enseignement et de recherche français ou étrangers, des laboratoires publics ou privés. 


\title{
Evolution des extrêmes hydrométriques en France à partir de données observées
}

\author{
Observed changes in hydrological extremes in France
}

\author{
Benjamin Renard ${ }^{(1)}$, Michel Lang ${ }^{(1)}$, Philippe Bois ${ }^{(2)}$, Anne Dupeyrat ${ }^{(3)}$, Olivier Mestre $^{(4)}$, Hélène Niel ${ }^{(5)}$ \\ Joël Gailhard $^{(6)}$, Carine Laurent ${ }^{(3)}$, Luc Neppel ${ }^{(5)}$, Eric Sauquet ${ }^{(1)}$ \\ (1) CEMAGREF Lyon, 3 bis quai Chauveau, 69009 Lyon \\ Tél : +33(0)4 722087 64, Fax : +33(0)4 784778 75, e-mail : renard@lyon.cemagref.fr \\ (2) Laboratoire d'étude des Transferts en Hydrologie et Environnement, 1025 rue de la piscine, \\ 38400 Saint Martin d'Hères \\ (3) EDF LNHE, 6 quai Watier, 78400 Chatou \\ (4) Météo-France / ENM, 42 avenue Gaspard Coriolis, 31057 TOULOUSE Cedex 1 \\ (5) HydroSciences, 300 avenue du Professeur Emile Jeanbrau, 34090 Montpellier \\ (6) EDF DTG, 21 avenue de l'Europe, 38040 Grenoble Cedex 9
}

Impact of climate change on hydrological extremes in France is still an open question. Results of a PNRH project about detecting trends or step-changes in the extreme hydrological regimes in France are here presented. A set of 192 hydrological series has been used, with at least 40 years of data and good quality rating curves. Statistical tests were applied on hydrological variables describing high flows (annual maxima, peak-over-threshold discharges and events frequency) and low flows (annual minima, duration and volume of droughts). Concerning high flows, no evidence of a consistent change has been found. The significant increase in the damages induced by floods thus arises from an increase in the vulnerability, caused by an unreasonable urbanization. Trends in annual minima may be stronger, but additional studies are needed in order to differentiate anthropogenic, metrological and climatic factors. Moreover, a Bayesian framework has been constructed in order to incorporate the hypothesis of stationarity as a new uncertainty in floods or droughts frequency analysis.

\section{I 口 INTRODUCTION}

«De toute évidence, le climat de la Terre a évolué à l'échelle régionale et mondiale depuis l'époque préindustrielle ». Ce constat émane du dernier rapport de synthèse du groupe d'experts intergouvernemental sur l'évolution du climat [IPCC, 2001]. Ce qui n'était encore qu'une hypothèse il $\mathrm{y}$ a une décennie semble aujourd'hui être un fait avéré : l'augmentation de la concentration des gaz à effet de serre dans l'atmosphère a eu des répercussions sur un certain nombre de variables climatiques. La température mondiale moyenne a ainsi augmenté d'environ $0,6{ }^{\circ} \mathrm{C}\left( \pm 0,2{ }^{\circ} \mathrm{C}\right)$ depuis 1900 , entraînant une augmentation du nombre de jours chauds $\left(T_{X} \geq 25^{\circ} \mathrm{C}\right)$ et une diminution du nombre de jours de gel $\left(T_{N} \leq 0^{\circ} \mathrm{C}\right)$. Les précipitations seraient elles aussi en augmentation, au moins dans l'hémisphère Nord, tandis que la couverture neigeuse et l'étendue des glaciers non polaires seraient en régression prononcée. Concernant la France, des résultats détaillés ont été fournis par les acteurs du projet IMFREX (http://medias.dsi.cnrs.fr/imfrex/web/projet/index). Au delà de ces observations directes, les modèles de circulation générale (GCM) prévoient tous une persistance de ces tendances au cours de ce siècle. Cependant, les incertitudes qui pèsent sur ces projections sont très importantes : les résultats peuvent varier significativement d'un GCM à l'autre, car un certain nombre de phénomènes, agissant à des échelles intermédiaires, sont ignorés dans les codes de calculs (dont la résolution spatiale est assez grossière) et restent parfois largement méconnus. De plus, les modèles sont conditionnés par des scénarios sociaux-économiques 
imaginés pour les années à venir. Ainsi, à l'horizon 2100, l'augmentation de la température globale devrait se situer entre 1.4 et $5.8^{\circ} \mathrm{C}$

Ces perturbations climatiques devraient avoir des répercussions sur le régime hydrologique. Cependant, ces conséquences sont difficiles à évaluer, pour deux principales raisons : tout d'abord, un réchauffement global peut avoir des effets antagonistes sur les écoulements, en augmentant à la fois l'évapotranspiration et les précipitations. De plus, les échelles spatiales et temporelles utilisées par les GCM (typiquement, plusieurs centaines de kilomètres de résolution horizontale au pas de temps journalier) ne concordent pas avec celles des modèles hydrologiques pluie/débit. Toutefois, les études publiées sur ce sujet [Kharin and Zwiers, 2000 ; Voss et al., 2002] prédisent une aggravation des phénomènes extrêmes, en basses eaux comme en hautes eaux. A ce jour, aucun signal cohérent à grande échelle n'a cependant été détecté pour les écoulements, malgré des changements avérés à l'échelle régionale (voir Lang et al. [2003] pour une brève revue).

Parallèlement au contexte du changement global, la vulnérabilité face aux évènements hydrologiques extrêmes est en constante augmentation, notamment en France. Une urbanisation mal maîtrisée a conduit la population à être de plus en plus exposée au risque d'inondation, tandis que la ressource en eau est de plus en plus sollicitée pour les besoins de l'agriculture et de l'industrie. Ces dernières années, la multiplication et la médiatisation de tels évènements, comme les inondations de décembre 2003 ou la canicule de l'été 2003, ont conduit à la perception d'une recrudescence des évènements extrêmes en France, qui n'est à ce jour pas établie.

L'absence de réponse claire de la part des scientifiques quant à la réalité d'un changement climatique en France est en partie due à la difficulté méthodologique de détecter des tendances dans des séries hydro-météorologiques qui, par nature, sont soumises à une importante variabilité naturelle. Il est toutefois primordial de pouvoir répondre à cette interrogation, car en matière de prévention des risques, la stationnarité des phénomènes dans le temps est une hypothèse qui est presque systématique.

Le travail présenté ici comporte trois objectifs majeurs : tout d'abord, progresser sur la méthodologie de détection de changements dans des séries hydro-météorologiques ; ensuite, donner une réponse quant à l'évolution du régime hydrologique des extrêmes en France; enfin, proposer des outils de gestion des risques dans un contexte non stationnaire.

\section{Q MÉTHODOLOGIE POUR LA DÉTECTION DE CHANGEMENTS}

\section{II.1 Présentation des principales approches}

La recherche de changements brusques ou graduels dans des séries statistiques est une préoccupation assez ancienne, qui a conduit à une grande abondance de méthodes disponibles dans la littérature. Les principaux facteurs influençant le choix d'une méthode concernent la structure probabiliste des variables (distribution, présence d'autocorrélation, données univariées ou multivariées), la taille des échantillons à analyser et le type de changement recherché (tendance, rupture unique, rupture multiple, changement en moyenne, en variance). On peut classer les différentes méthodes disponibles en quatre grandes familles:
Les approches descriptives : il s'agit de représenter visuellement une série de données afin de mettre en avant son comportement vis-à-vis de l'hypothèse de stationnarité, soit par simple visualisation du graphique des valeurs chronologiques, soit par des méthodes plus sophistiquées utilisant le lissage ou des approches descriptives multivariées (analyse de données).

Les procédures de segmentation et d'homogénéisation [Hubert et al., 1989; Peterson et al., 1998; Mestre, 2000 ; Kehagias, 2004] consistent à segmenter une série en plusieurs sous-séries stationnaires. Elles sont en général dédiées à la recherche de ruptures multiples, d'origine métrologique notamment, bien qu'elles aient aussi été appliquées à des études climatiques, dans le cas de phénomènes présentant une variabilité à l'échelle de la dizaine d'années [Hubert et al., 1989].

Les tests statistiques, qui permettent d'évaluer la significativité d'une hypothèse. Ils sont en général assez simples à mettre en place et sont donc particulièrement adaptés à une étude sur un grand nombre de stations. La littérature fournit une grande variété de tests de détection de changements, qui couvrent la quasi-totalité des situations que nous rencontrerons.

L'approche Bayésienne: ce cadre d'analyse permet de combiner facilement plusieurs modèles ou niveaux d'analyse et présente un intérêt certain pour l'analyse régionale de changements.

La première approche a été utilisée dans ce projet à titre d'analyse préliminaire, suivie ensuite par l'application de tests statistiques à l'échelle locale. L'approche par segmentation n'a pas été retenue car le niveau de significativité des résultats n'est pas facilement accessible hors du cadre Gaussien.

\section{II.2 Analyse comparative de la puissance de tests statistiques}

De nombreuses études ont déjà été effectuées pour le choix d'un test adapté à une situation donnée [Lubès-Niel et al., 1998 ; Kundzewicz and Robson, 2000 ; Lemaitre, 2002 ; Dindar, 2003 ; Kundzewicz, 2004 ; Kundzewicz and Robson, 2004 ; Lang et al., 2004 ; Zhang et al., 2004], mais leurs conclusions ne sont pas toujours cohérentes. Par exemple, Kundzewicz et Robson [2004] conseillent d'utiliser des tests non paramétriques car ils sont presque aussi puissants que les tests paramétriques, tout en étant très souples d'utilisation. A l'inverse, Zhang et al. [2004] recommandent plutôt d'utiliser des tests paramétriques adaptés aux lois des valeurs extrêmes, du fait d'un net gain de puissance.

Nous avons repris ces analyses afin de définir un schéma de sélection du test statistique adapté au problème de détection dans le régime des extrêmes hydrométriques. Une série de simulations Monte-Carlo a permis d'étudier la puissance des tests (aptitude à détecter correctement un changement sur une série) et leur robustesse face au non respect d'hypothèses sur les valeurs de l'échantillon (normalité, indépendance). Il en ressort que les tests paramétriques basés sur le rapport de vraisemblance de deux hypothèses alternatives sont les plus performants (tests LR), sous réserve que les hypothèses de base soient bien adaptées au cas des valeurs extrêmes. Ce type de test, qui n'a été utilisé que récemment pour la détection des évolutions liées au changement climatique [Coles, 2001 ; Mestre, 2003 ; Zhang et al., 2004; Parey et al., 2006], a été adapté au cas de la détection de tendances ou de ruptures dans les paramètres de position et d'échelle 
des lois de valeurs extrêmes [Renard, 2006]. Par ailleurs, une procédure de sélection de tests a été établie (fig. 1), en tenant compte de l'existence d'autocorrélation, du type de distribution, du type de changement attendu et de la longueur de l'échantillon.

\section{ANALYSE DE STATIONNARITE EN FRANCE MÉTROPOLITAINE}

\section{III.1 Présentation des données}

192 longues séries de débits journaliers, ayant plus de 40 ans d'ancienneté, ont été sélectionnées sur l'ensemble de la France métropolitaine. La qualité de ces données a été évaluée par une visite aux différents gestionnaires (DIREN, $\mathrm{EDF}, \mathrm{CNR})$. Ces stations sont réputées pas ou faiblement influencées en crues, ou sont des débits naturels reconstitués (débit calculé qui passerait au droit de la station en l'absence des influences principales). Par contre, seule une partie de ces séries pourra être utilisée en étiage, les basses eaux subissant plus fréquemment des influences directes (prélèvements, soutien d'étiage, ...).

Du point de vue hydrologique, l'ensemble des régimes présents en France métropolitaine est représenté dans l'échantillon de stations, des régimes pluviaux océaniques ou méditerranéens aux régimes nivaux ou glaciaires, en passant par les différents régimes mixtes. De même, des bassins versants de toutes tailles sont représentés (de 5 à $109930 \mathrm{~km}^{2}$, médiane $915 \mathrm{~km}^{2}$ ).

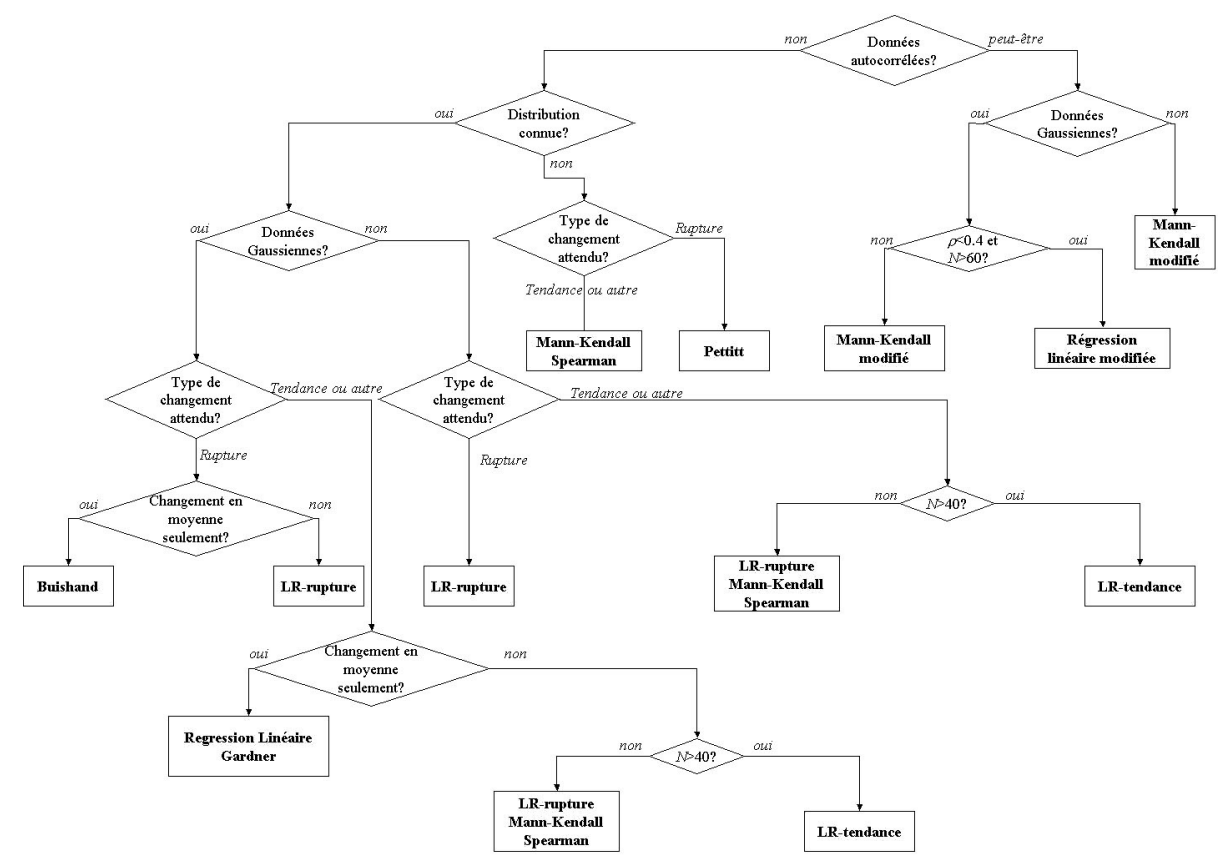

Figure 1 : Schéma de sélection d'un test de stationnarité adapté aux caractéristiques probabilistes de la variable étudiée.
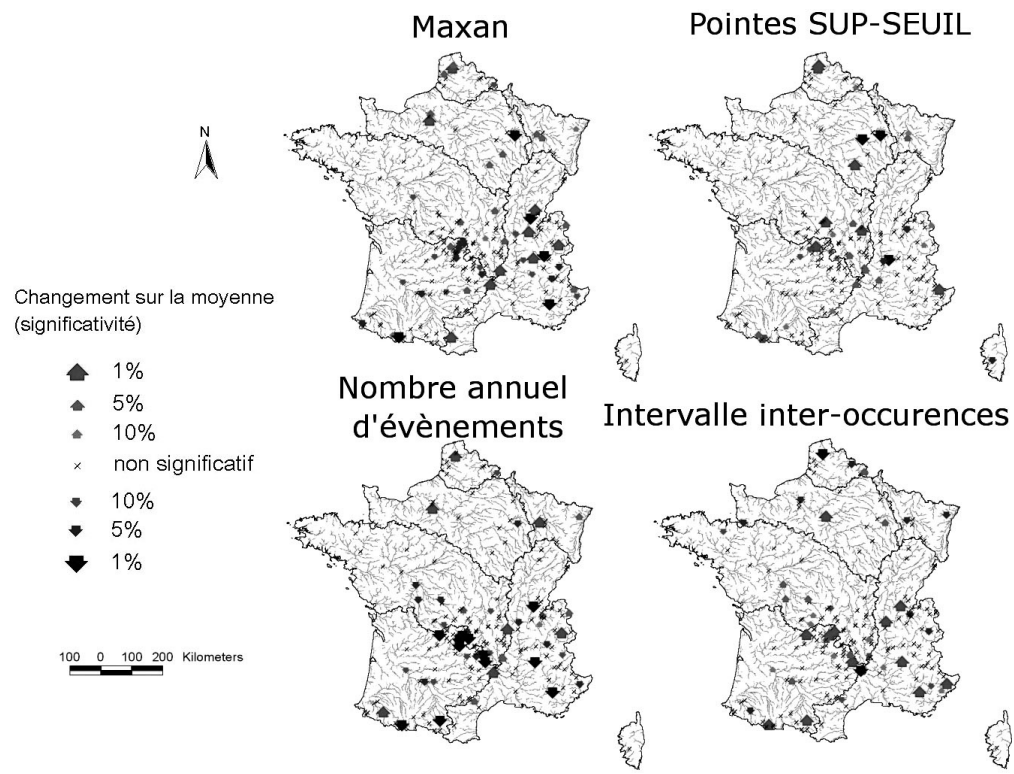

Figure 2 : Tendances détectées sur les variables de crue (voir définitions page suivante). 
Tableau 1 : Tests de tendance utilisés pour les huit variables d'étude.

$L R=$ rapport de vraisemblance, $M K=$ Mann-Kendall, $M M K=$ Mann-Kendall modifié.

\begin{tabular}{cccc}
\hline Variable & Distributions & Autocorrélation $\rho$ & Test appliqué \\
\hline MAXAN & GEV & Non & LR \\
SUP-SEUIL & Pareto généralisée & Non & LR \\
Nombre annuel d'évènements & Poisson & Non & LR \\
Intervalle inter-occurrence & Exponentielle & Non & MMK si $\rho$ significatif, LR sinon \\
VCN30 & GEV & Doit être testée & MMK si $\rho$ significatif, LR sinon \\
QCN30 & GEV & Doit être testée & MMK si $\rho$ significatif, MK sinon \\
Déficit de volume & Inconnue & Doit être testée & MMK si $\rho$ significatif, MK sinon \\
\hline
\end{tabular}

A partir des chroniques journalières, des variables descriptives des hautes et des basses eaux ont été extraites. Nous nous limitons ici aux résultats concernant huit variables. Le maximum annuel (MAXAN), les pointes de débits supérieurs à un seuil (SUP-SEUIL), le nombre annuel d'évènements supérieurs à un seuil et les durées entre deux évènements successifs (intervalles inter-occurrences) décrivent les crues, tandis que le minimum annuel des débits moyens sur 30 jours (VCN30), le minimum annuel des débits seuils sur 30 jours (QCN30), le déficit annuel de volume par rapport à un seuil de bas débit et le nombre de jours passés chaque année sous un seuil de bas débit (durée) décrivent les basses eaux. De plus, l'année est considérée ici au sens hydrologique, c'est à dire que l'année hydrologique débute pour les crues (resp. les étiages) au mois ayant le plus faible (resp. le plus fort) débit mensuel inter-annuel.

Compte tenu du schéma de sélection de test qui a été réalisé, chacune de ces huit variables a été traitée avec le test de tendance le plus adapté. Le Tableau 1 résume les choix effectués. Nous avons supposé que les variables de crue ne présentaient pas d'autocorrélation, ce qui semble être une hypothèse physiquement raisonnable dans la quasi-totalité des bassins versants étudiés. Inversement, les variables d'étiage peuvent posséder une mémoire inter-annuelle, liée à l'inertie du mécanisme remplissage/vidange de la nappe. Nous avons donc testé la significativité de ces autocorrélations, et utilisé le cas échéant des tests adaptés.

\section{III.2 Résultats}

La Figure 2 présente les évolutions détectées sur les variables relatives aux hautes eaux. L'intensité des crues (valeurs maximales annuelles ou supérieures à un seuil) ne présente pas de tendance généralisée à la hausse, contrairement à ce que pourrait laisser supposer l'augmentation significative des dommages dus aux inondations. Ce phénomène provient bien de l'augmentation de la vulnérabilité aux inondations, comme indiqué en introduction. Le nombre d'événements de crue aurait même eu tendance à diminuer sur un nombre non négligeable de stations (et par voie de conséquence une augmentation du délai entre deux événements successifs sur ces stations).

Pour les étiages (fig. 3), seules 125 chroniques réputées non influencées ou reconstituées sont utilisées. Les changements significatifs semblent plus nombreux sur le débit minimal (VCN30 et QCN30). Les tendances affectant le volume ou la durée des étiages semblent par contre moins nombreuses. Dans tous les cas, il semble encore une fois difficile de dégager une cohérence hydrologique de ces résultats, ce qui incite à la prudence avant d'attribuer ces changements à une cause climatique.

Des investigations complémentaires sont donc nécessaires pour interpréter les changements à la hausse ou à la baisse et apprécier ce qui relève des influences anthropiques (ouvrages de soutien d'étiage, prélèvement d'eau), des problè-

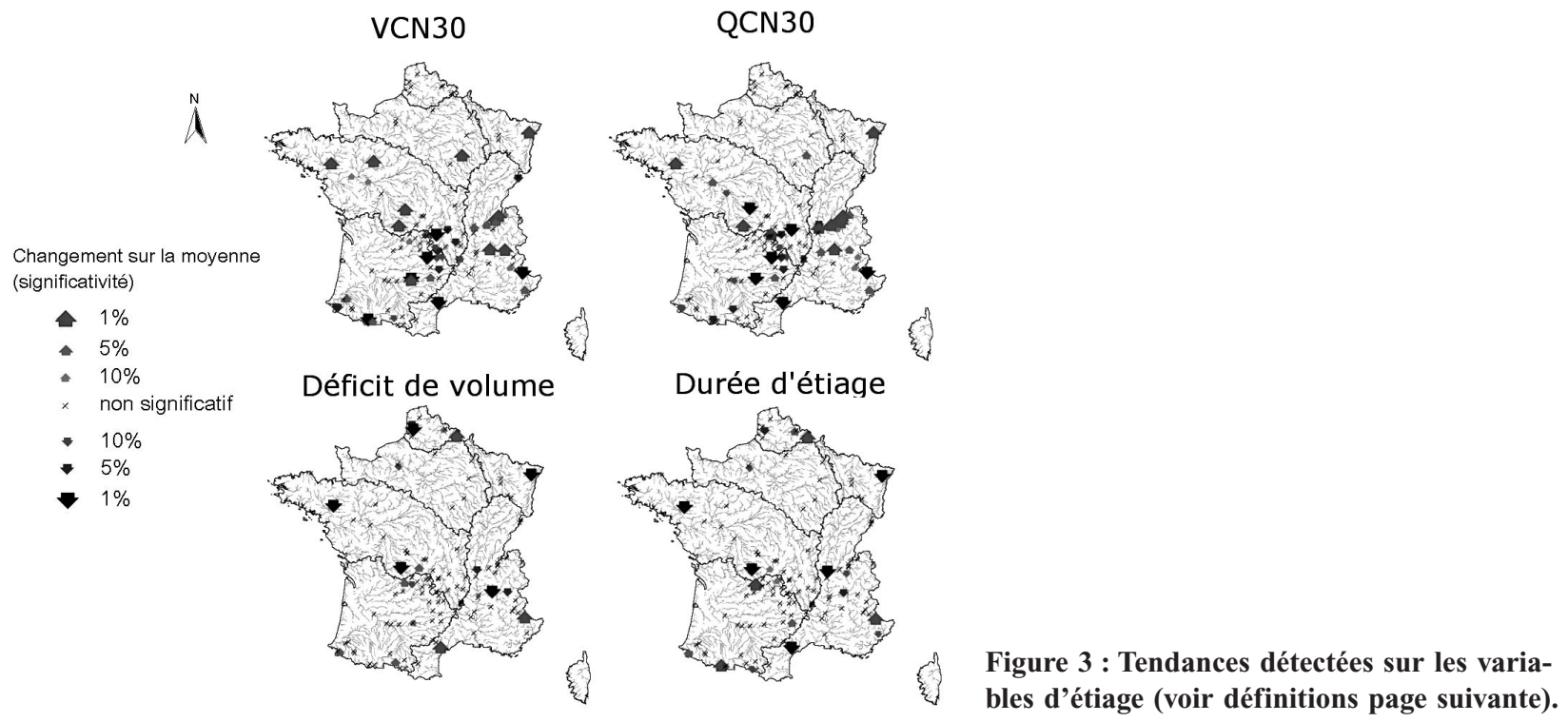


mes métrologiques (changement de courbe de tarage) et des influences climatiques (précipitations et températures). Nous avons donc entrepris un nouveau dialogue avec les gestionnaires, afin de critiquer les changements détectés. Plus précisément, des tests de rupture ont également été effectués, ce qui permet d'estimer une date de rupture approximative. Nous recherchons ensuite dans l'historique des stations de mesure des évènements ayant pu conduire aux ruptures détectées. Ce travail est toujours en cours, il est donc impossible de donner des conclusions définitives. Cependant, dans un nombre non négligeable de cas, nous avons pu relier les changements détectés sur les variables de crue à un changement d'extrapolation de la courbe de tarage. Pour les étiages, ce travail de critique est plus délicat, car certaines influences peuvent être suspectées mais ne sont pas facilement quantifiables (prélèvements pour l'irrigation, par exemple).

\section{ANALYSE FRÉQUENTIELLE NON STATIONNAIRE}

\section{IV.1 Principe de l'approche Bayésienne}

Un modèle Bayésien d'analyse a été développé [Renard et al., 2006a] pour sortir de la simple alternative qui consiste soit à accepter soit à rejeter l'hypothèse de stationnarité. Nous ne présentons ici que les principes généraux d'une telle analyse. Tout d'abord, quatre étapes sont nécessaires pour mettre en place une analyse Bayésienne :

1. Choix de la distribution des observations: comme dans le cadre de l'analyse fréquentielle classique, nous supposons que les données $\mathbf{X}$ suivent une loi paramétrée par un vecteur $\theta$. Sous l'hypothèse d'indépendance des observations, il est alors possible de calculer la vraisemblance du vecteur $\boldsymbol{X}$, que nous noterons $p(\boldsymbol{X} \mid \boldsymbol{\theta})$

2. Spécification de la distribution a priori des paramètres: Une des caractéristiques fondamentales de l'analyse Bayesienne est que $\theta$ n'est pas considéré comme un vecteur constant, dont nous souhaitons estimer la « vraie» valeur, mais comme une variable aléatoire, dont nous cherchons à connaître les caractéristiques. Une première étape consiste à traduire des connaissances exogènes aux données que nous souhaitons intégrer à l'analyse sous la forme d'une distribution $\pi(\theta)$. A titre d'exemple, une analyse régionale ou des connaissances empiriques peuvent être utilisées pour construire cette distribution.

3. Application du théorème de Bayes: la distribution a posteriori des paramètres, $p(\theta \mid \boldsymbol{X})$, est calculée par la formule suivante :

$$
p(\theta \mid \boldsymbol{X})=\frac{\pi(\theta \mid) p(\boldsymbol{X}|\boldsymbol{\theta}|)}{\int \pi(\boldsymbol{\theta} \mid) p(\boldsymbol{X}|\boldsymbol{\theta}|) d \theta \mid}
$$

4. Utilisation de la distribution a posteriori: l'inférence ne se limite pas ici à une valeur estimée suivant un critère prédéfini, mais à la distribution complète des quantités à estimer (paramètres du modèle ou quantiles, par exemple).

La démarche que nous avons adoptée, inspirée par le travail de Perreault et al. [2000a; 2000b], consiste à mettre en concurrence plusieurs modèles : un modèle stationnaire, et un (ou plusieurs) modèle(s) dont les paramètres dépendent du temps. Il est alors possible de calculer la probabilité $a$ posteriori de chaque modèle, de la manière suivante :

$$
P\left(M_{k} \mid \boldsymbol{X}\right)=\frac{p\left(\boldsymbol{X} \mid M_{k}\right) \pi\left(M_{k}\right)}{\sum_{j=1}^{q} p\left(\boldsymbol{X} \mid M_{j}\right) \pi\left(M_{j}\right)},
$$

où $\pi\left(M_{k}\right)$ est la probabilité a priori du modèle $k$, et $p\left(\boldsymbol{X} \mid M_{k}\right)$ est la vraisemblance marginale des observations dans le modèle $k$, définie par:

$$
p\left(\boldsymbol{X} \mid M_{k}\right)=\int p\left(\boldsymbol{X}, \theta \mid M_{k}\right) d \theta=\int p\left(\boldsymbol{X} \mid \theta, M_{k}\right) \pi\left(\theta \mid M_{k}\right) d \theta
$$

Il est alors possible d'évaluer une distribution a posteriori des quantiles de crue, qui prend en compte à la fois l'incertitude d'échantillonnage et l'incertitude liée au choix d'un modèle (stationnaire, tendance linéaire, quadratique, rupture, ...), de la manière suivante :

Répéter $N$ fois :

- Tirage au sort d'un modèle $M^{*}$, avec les probabilités $p\left(M_{1} \mid X\right), \ldots, p\left(M_{k} \mid X\right)$.

- Echantillonnage d'un vecteur de paramètres $\theta^{*}$ à partir de $p\left(\theta \mid X, M^{*}\right)$.

- Calcul de $q_{p}(t)$, le quantile de probabilité $p$ calculé dans le modèle $M^{*}$ avec les paramètres $\theta^{*}$ au temps $t$.

L'échantillon de valeurs obtenu peut alors être utilisé pour estimer la distribution des quantiles. Notons enfin que la plupart des formules décrites ci-dessus ne peuvent en général pas être évaluées explicitement, ce qui nécessite de recourir à des méthodes de Monte Carlo par Chaînes de Markov (MCMC). Un travail spécifique a été effectué récemment par Garreta [2005] et Renard et al. [2006b] sur cet aspect.

\section{IV.2 Cas d'étude}

Nous avons appliqué la méthode présentée ci-dessus aux crues supérieures à $23 \mathrm{~m}^{3} \mathrm{~s}^{-1}$ depuis 1907 sur la Drôme à Luc en Diois $\left(194 \mathrm{~km}^{2}\right)$. Ce type d'échantillonnage conduit à s'intéresser à la fois au processus d'occurrence et à l'intensité des crues (fig. 4). Nous avons donc utilisé les modèles suivants :

Processus d'occurrence: les intervalles inter-occurrences suivent une loi exponentielle. Le paramètre de cette loi est soit stationnaire (modèle $M_{0}^{\exp }$ ), soit linéairement dépendant du temps (modèle $M_{1}^{\text {exp }}$ ).

Intensité : Les pointes de crue sélectionnées suivent une loi de Pareto généralisée. Le paramètre d'échelle de cette loi est soit stationnaire (modèle $M_{0}^{G P D}$ ), soit linéairement dépendant du temps (modèle $M_{1}^{G P D}$ ).

Pour chacun de ces processus, en supposant que les deux modèles sont a priori équiprobables, nous obtenons les probabilités $a$ posteriori suivantes :

$$
\text { Processus d'occurrence: } \quad p\left(M_{0}^{\exp } \mid X\right)=0,11 \text {, }
$$
$p\left(M_{1}^{\exp } \mid X\right)=0,89$, la fréquence des évènements semble donc non stationnaire (en l'occurrence, elle semble décrô̂tre). 


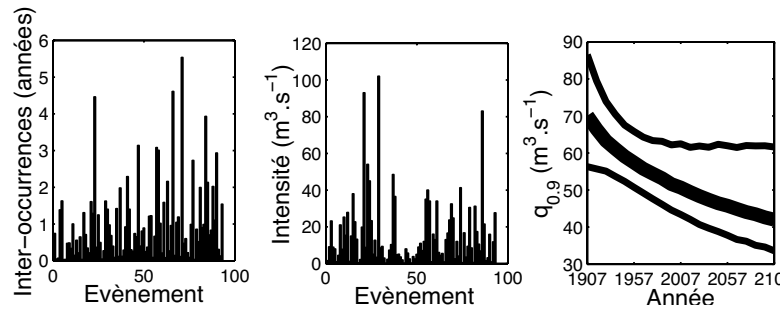

Figure 4 : (a) série des intervalles inter-occurrences, (b) série des intensités, (c) trajectoire de la crue décennale au cours du temps, et intervalle de confiance à $90 \%$.

Intensité : $p\left(M_{0}^{G P D} \mid X\right)=0,79, p\left(M_{1}^{G P D} \mid X\right)=0,21,1$ 'intensité des évènements semble stationnaire.

La trajectoire de la crue décennale au cours du temps est représentée dans la Figure 4. L'intervalle de confiance à $90 \%$ de la loi a posteriori du quantile, représenté en trait fin, prend en compte les incertitudes liées à l'échantillonnage et à la stationnarité des processus.

\section{CONCLUSIONS ET PERSPECTIVES}

Le travail effectué a permis de progresser sur le plan méthodologique de la détection de tendances et de ruptures dans le régime des extrêmes hydrométriques. Une procédure de sélection de tests statistiques a été établie en tenant compte du contexte particulier des valeurs extrêmes dans les séries hydrométriques. Un cadre d'analyse Bayésienne a ensuite été développé pour permettre une analyse probabiliste des séries chronologiques dans un contexte non stationnaire. Les premiers résultats obtenus sur un jeu d'environ 200 longues séries hydrométriques françaises ne montrent pas de tendance uniforme sur les crues ou sur les étiages. Ils sont en cours d'interprétation pour faire la part des changements dus à des problèmes de métrologie ou à des effets anthropiques directs (aménagements, prélèvements) non relevés a priori. Dans un second temps, les tendances ou ruptures non expliquées seront mises en relation avec la climatologie et les processus physiques de transformation des pluies en débit. Enfin, nous espérons pouvoir étendre les résultats obtenus localement à l'échelle régionale, que ce soit du point de vue de la détection de changements ou de l'analyse fréquentielle non stationnaire.

\section{Q REMERCIEMENTS}

Ce travail a été financé par le PNRH entre 2002 et 2004. Le travail de thèse de $\mathrm{B}$. Renard est soutenu financièrement par EDF et le CEMAGREF. Nous remercions le MEDD pour nous avoir permis d'utiliser les données de la banque HYDRO, ainsi que l'ensemble des gestionnaires de l'hydrométrie qui nous ont toujours accueillis avec sympathie.

\section{BIBLIOGRAPHIE}

[1] Coles, S. (2001). - An Introduction to Statistical Modeling of Extreme Values, Springer Series in Statistics, $210 \mathrm{p}$.
[2] Dindar, L. (2003). - Analyse comparative de la puissance de tests de détection de ruptures dans des séries chronologiques de débit, 84 p. Rapport de stage, CEMAGREF.

[3] Garreta, V. (2005). - Analyse Bayésienne des débits de crue: méthodes MCMC et cas d'études, $97 \mathrm{pp}$. Rapport de stage, CEMAGREF.

[4] Hubert, P., J.-P. Carbonnel, and A. Chaouche, (1989). - Segmentation des séries hydrométéorologiques Application à des séries de précipitations et de débits de l'Afrique de l'Ouest. J. Hydrol., 110, pp. 349-367.

[5] IPCC (2001). - Climate change 2001 : synthesis report.

[6] Kehagias, A. (2004). - A hidden Markov model segmentation procedure for hydrological and environmental time series. Stoch. Environ. Res. Risk Assess., 18, pp. 117-130.

[7] Kharin, V. V. and F. W. Zwiers, (2000). - Changes in the extremes in an ensemble of transient climate simulations with a coupled atmosphere-ocean GCM. J. Clim., 13, pp. 3760-3788.

[8] Kundzewicz, Z. W. (2004). - Searching for change in hydrological data. Hydrol. Sci. J.-J. Sci. Hydrol., 49, pp. 3-6.

[9] Kundzewicz, Z. W. and A. J. Robson (2000). - Detecting trend and other changes in hydrological data. Rapport WMO, $160 \mathrm{p}$.

[10] Kundzewicz, Z. W. and A. J. Robson (2004). — Change detection in hydrological records - a review of the methodology. Hydrol. Sci. J.-J. Sci. Hydrol., 49, pp. 7-19.

[11] Lang, M., P. Bois, O. Mestre, H. Niel, and E. Sauquet, (2003). - Détection de changements éventuels dans le régime des crues - Rapport de première année, projet PNRH., 75 p.

[12] Lang, M., B. Renard, L. Dindar, F. Lemaitre, and P. Bois, (2004). - Use of a statistical test based on Poisson process for the detection of changes in peak-over-threshold series. Hydrology: Science \& Practice for the 21st Century, London, pp. 158-164.

[13] Lemaitre, F. (2002). - Recensement des tests de détection de tendances ou de ruptures adaptés à l'analyse de stationnarité des régimes de crues en France. Rapport de fin d'étude TPE, 94 p. CEMAGREF.

[14] Lubės-Niel, H., J. M. Masson, J.-E. Paturel, and E. Servat, (1998). - Variabilité climatique et statistiques. Etude par simulation de la puissance et de la robustesse de quelques tests utilisés pour vérifier l'homogénéité de chroniques. Rev. Sci. Eau, 3, pp. 383-408.

[15] Mestre, O. (2000). - Méthodes statistiques pour l'homogénéisation de longues séries climatiques, Laboratoire de statistiques et probabilités, Université Paul Sabatier, Mémoire de thèse, $229 \mathrm{p}$.

[16] Mestre, O. (2003). - Modélisation des paramètres de la GEV: application à l'étude des crues. $X V I^{e}$ entretiens du centre Jacques Cartier, Lyon.

[17] Parey, S., F. Malek, , C. Laurent, and D. DacunhaCastelle, (2006). - Trends and climate evolution: statistical approach for very high temperatures in France. submitted to Climatic Change.

[18] Perreault, L., J. Bernier, B. Bobee, and E. Parent, (2000a). - Bayesian change-point analysis in hydrometeorological time series. Part 1 . The normal model revisited. $J$. Hydrol., 235, pp. 221-241.

[19] Perreault, L., J. Bernier, B. Bobee, and E. Parent, (2000b). - Bayesian change-point analysis in hydrometeorological time series. Part 2. Comparison of change-point models and forecasting. J. Hydrol., 235, 242-263.

[20] Peterson, T. C., D. R. Easterling, T. R. Karl, P. Groisman, N. Nicholls, N. Plummer, S. Torok, I. Auer, R. Boehm, 
D. Gullett, L. Vincent, R. Heino, H. Tuomenvirta, O. Mestre, T. Szentimrey, J. Salinger, E. J. Forland, I. Hanssen-Bauer, H. Alexandersson, P. Jones, and D. PARKER, (1998). - Homogeneity adjustments of in situ atmospheric climate data: A review. Int. J. Climatol., 18, pp. 1493-1517.

[21] Renard, B. (2006). - Détection et prise en compte d'éventuels impacts du changement climatique sur les extrêmes hydrologiques en France, 364 p. Thèse de Doctorat, INPG, Cemagref.

[22] Renard, B., M. Lang, and P. Bois, (2006a). - Statistical analysis of extreme events in a non-stationary context via a Bayesian framework. Stoch. Environ. Res. Risk Assess. Under press.
[23] Renard, B., V. Garreta, and M. LANG, (2006b). - An empirical comparison of MCMC methods used in Bayesian inference. Application for regional trend estimation. submitted to Water Resources Research.

[24] Voss, R., W. May, and E. Roeckner, (2002). — Enhanced resolution modelling study on anthropogenic climate change: Changes in extremes of the hydrological cycle. Int. J. Climatol., 22, pp. 755-777.

[25] Zhang, X. B., F. W. Zwiers, and G. L. Li, (2004). - Monte Carlo experiments on the detection of trends in extreme values. J. Clim., 17, pp. 1945-1952. 